\title{
Multilinguales
}

\section{Des femmes qui voyagent}

$$
\text { نساء في ترحال }
$$

\section{Denise Brahimi}

\section{(2) OpenEdition}

\section{Journals}

\section{Édition électronique}

URL : http://journals.openedition.org/multilinguales/335

DOI : 10.4000/multilinguales.335

ISSN : 2335-1853

\section{Éditeur}

Université Abderrahmane Mira - Bejaia

\section{Référence électronique}

Denise Brahimi, «Des femmes qui voyagent », Multilinguales [En ligne], 8 | 2017, mis en ligne le 01 juin 2017, consulté le 17 septembre 2019. URL : http://journals.openedition.org/multilinguales/335 ; DOI : 10.4000/multilinguales.335

Ce document a été généré automatiquement le 17 septembre 2019.

\section{(c) (i) (9)}

Multilinguales est mise à disposition selon les termes de la Licence Creative Commons Attribution -

Pas d'Utilisation Commerciale - Pas de Modification 4.0 International 


\title{
Des femmes qui voyagent
}

$$
\text { نساء في ترحال }
$$

\author{
Denise Brahimi
}

\section{Des femmes et des ecrivaines}

1 Pourquoi ne pas dire plus simplement : des femmes voyageuses?

2 Parce que les trois femmes dont il sera question ici, Flora Tristan, George Sand et Isabelle Eberhardt ne sont pas de celles qu'on pourrait appeler des voyageuses professionnelles, une catégorie bien représentée et qu'on peut inventorier à partir d'un certain nombre de critères permettant (peut-être) un classement. Ce sont des femmes que les circonstances ont amenées à voyager (Flora Tristan), ou qui ont réfléchi aux particularités du voyage dans la vie des femmes (George Sand), ou qui ont «nomadisé » faute de pouvoir supporter la vie sédentaire (Isabelle Eberhardt). Par ailleurs il est évidemment intéressant de choisir des femmes qui sont aussi ou surtout des écrivaines, et qui ont la capacité d'écrire, ce qui est une autre manière de dire, de réfléchir, d'analyser et de transmettre - car il y faut à la fois le désir et la capacité. C'est évidemment un avantage très précieux pour qui souhaite s'engager, au moins intellectuellement, dans cette thématique. Ceci dit, on peut peut-être remarquer d'emblée que le mot "intellectuellement » est trop restrictif, sans doute pour les chercheuses qui se sentent concernées par ce sujet mais d'abord et avant tout pour les femmes auxquelles elles s'intéressent, c'est-à-dire, dans le cadre de notre réflexion, Flora Tristan, George Sand et Isabelle Eberhardt ${ }^{1}$.

1/Flora Tristan n'aurait sans doute pas voyagé si certaines circonstances de sa vie ne l'y avaient obligée. A quoi il faut ajouter qu'elle aurait pu néanmoins exclure toute idée de voyage au long cours, au nom du fait que c'était absolument impossible pour une femme comme elle. Mais ce n'est pas ainsi qu'elle a réagi et c'est d'abord pour cela qu'elle nous intéresse. Faute de pouvoir revenir en détail sur l'histoire de sa vie, on s'en tiendra principalement ici à ce qu'on apprend de sa brève destinée (1803-1844) en lisant son maître livre, Pérégrinations d'une paria (1979). 
4 Le livre, paru en 1838, raconte le voyage au Pérou fait par son auteure en 1833. Avant cette dernière date, Flora Tristan avait rencontré nombre de difficultés mais les avait vécues sur un mode personnel sans les inclure dans une préoccupation sociale et collective.

5 Si elle part au Pérou, c'est pour s'y faire reconnaître comme fille de son père, un général péruvien mort lorsque Flora n'avait que cinq ans, et pour tenter de récupérer l'héritage lié à cette paternité. De ces deux motivations, c'est certainement la première qui a été pour elle la plus importante. Flora Tristan (et c'est un point qu'elle a en commun aussi bien avec George Sand qu'avec Isabelle Eberhardt, les deux autres femmes dont le rapport au voyage sera ici évoqué), souffre de ce qu'on pourrait définir comme une sorte de bâtardise sociale, à condition de prendre le mot au sens large.

\section{Bâtardise sociale}

6 Que signifie cette formule dans son cas et en quoi s'agit-il d'une situation qui a pu jouer un rôle fort dans son étonnante décision d'affronter l'aventure du voyage? Flora Tristan est née en 1802 d'un père de haut lignage qui était général dans l'armée péruvienne. Malheureusement ce père meurt cinq ans plus tard sans avoir pris soin de faire reconnaître officiellement Flora pour sa fille, si bien qu'en dépit d'un mariage religieux Madame Tristan se retrouve sans argent, ce qui oblige la jeune Flora à connaître très tôt la pauvreté et le sort d'une ouvrière. Le fait important est qu'elle se révolte contre cette situation, dont on peut penser qu'elle lui est doublement et à tous égards insupportable. Le mot n'est pas trop fort si l'on en juge par les conséquences d'un état de fait, insupportable donc au point de la pousser à partir au Pérou en 1835. Savait-elle elle-même si son but principal était de récupérer son héritage auprès de son oncle et de sa famille péruvienne-ou plus encore d'être reconnue par eux comme la digne fille de son père ? Fille de général et pauvre ouvrière, telle est en tout cas la bâtardise sociale de Flora Tristan, incontestablement à l'origine de ses "pérégrinations".

7 D'un point de vue strictement factuel, on peut considérer que sa double tentative est un échec. Mais on pourrait dire que Flora a gagné bien davantage que ce qu'elle croyait chercher, du fait qu'elle a acquis non seulement l'expérience éminemment formatrice du voyage en tant que tel, ce qu'on pourrait appeler l'aventure existentielle du voyage, mais que de plus elle en a tiré tous les prolongements dont il pouvait suggérer l'idée à une femme généreuse comme elle, intelligente, passionnée et inventive. Qu'on en juge plutôt par le bref inventaire qui va suivre.

\section{Prolongements du voyage}

8 Dès 1835, Flora Tristan publie un premier livre, ou plutôt un opuscule de faible dimension qui est le résultat direct de ses observations de voyageuse occasionnelle. Il s'intitule Nécessité de faire un bon accueil aux femmes étrangères (1988) et il témoigne d'un grand intérêt pour les difficultés rencontrées par les femmes lorsqu'il leur faut voyager. De son expérience personnelle, Flora Tristan tire l'idée que pour la majorité des gens, les déplacements d'une femme seule sont sentis comme intolérables et ne peuvent valoir à la malheureuse que suspicion, mépris ou mauvais traitements, en tout cas, à coup sûr, mauvais accueil. C'est pourquoi elle veut fonder une association à but 
humanitaire destinée d'une part à assurer, de manière pragmatique et concrète, la protection des voyageuses et d'autre part à revendiquer politiquement le droit au voyage pour le genre féminin, comme nous dirions aujourd'hui. Le présentateur moderne de cette oeuvre, Denys Cuche, écrit à ce propos: "Revendiquer le droit au voyage pour les femmes revient donc, dans l'esprit de Flora, à promouvoir l'autonomie des femmes et à défendre leur participation à la vie publique » (1988: 37).

9 La publication de ce petit livre est la preuve que le voyage au Pérou a ouvert l'esprit de Flora Tristan aux préoccupations politiques et sociales. Mais un autre voyage, qu'elle fait en 1839, à Londres cette fois, a un rôle encore plus déterminant en ce sens. Elle en tire un livre, Promenades dans Londres (1978), paru en 1840, qui manifeste sa vocation de militante socialiste, grâce à l'analyse faite sur le terrain de ce qui est alors le prototype de la société industrielle. Il apparaît clairement que pour elle, cette expression signifie une société fondée sur l'exploitation du travail ouvrier.

10 Ainsi et bien qu'on ne puisse développer ici ce qu'elle fut par la suite, la vie de Flora Tristan $^{2}$ illustre parfaitement le rôle du voyage pour transformer une féministe romantique, farouchement individualiste, en militante totalement dévouée à la cause du prolétariat ouvrier. Flora Tristan est une femme qui a voyagé : on voit de quel poids se leste la formule : tribulations certes et pérégrinations autant qu'on voudra, mais à condition de ne pas cantonner ces mots dans un sens descriptif et anecdotique. Avec Flora Tristan, la femme cesse d'être assimilée globalement au rôle de femme au foyer et de symboliser une permanence qui serait l'autre nom de l'immobilité : «et pourtant elle bouge », cette formule nous rappelle les grandes découvertes qui ont ébranlé le socle des croyances archaïques profondément inscrites dans les mentalités.

11 2/George Sand elle-même n'a pas échappé à ce type de vision dans lequel certaines formules ont tendance à l'enfermer. Lorsqu'on la désigne comme « la bonne dame de Nohant ", on l'assimile à un lieu dans l'idée plus ou moins consciente de l'immobiliser, c'est-à-dire de réduire voire d'annihiler son caractère potentiellement subversif. Pourtant, ce n'est certainement pas l'idée qu'elle se fait d'elle-même et elle fait explicitement l'éloge du voyage dans l'un de ses premiers grands romans, Consuelo, de 1842. Il apparaît que la jeune femme qui donne son titre à la première partie de ce long roman est une figure d'elle-même (certes parmi beaucoup d'autres) et qu'elle se représente à travers son hérö̈ne comme une adepte du « voyage de formation »-celui qui permet d'entrer dans la pleine maturité.

\section{Vertus politiques et sociales du voyage}

12 Avec Consuelo (George Sand, 1959) ${ }^{3}$, on a la preuve que George Sand croyait aux vertus politiques et sociales du voyage pour les femmes en général et particulièrement pour les femmes artistes-une catégorie où elle ne peut manquer de se ranger elle-même, mais avec la volonté de dépasser son cas personnel pour atteindre à la généralité.

13 Consuelo, héroïne du roman, est née en Espagne vers le milieu du 18ème siècle mais vient bientôt en Italie avec sa mère, non sans passer par divers autres pays à la manière des Bohémiens ${ }^{4}$ auxquels elle s'apparente. Destinée par son talent et par les leçons d'un maitre éminent à devenir une cantatrice de génie, elle doit quitter Venise par suite d'amours malheureuses et trouve asile dans un château en Allemagne. Cependant, de là aussi il lui faut partir et elle se retrouve sur les routes en direction de Vienne, dans le même dénuement qu'elle a connu enfant avec sa mère. La rupture est brutale après le 
mode de vie très privilégié qu'elle avait au château et qui aurait pu lui donner le goût du luxe, en tout cas d'une installation confortable et sûre. Or, bien que consciente des dangers qui guettent une jeune fille comme elle, seule sur les routes en pays inconnu, elle a un sursaut de contentement à l'idée de renouer grâce au voyage avec ses origines plébéiennes (le mot est d'elle) :

Je suis bien folle et bien vaine, se dit-elle, si je ne puis réaliser ce que j'ai conçu. Eh quoi ! sera-t-il dit que la fille de ma mère se soit efféminée dans les douceurs de la vie, au point de ne pouvoir plus braver le soleil, la faim, la fatigue et les périls? J'ai fait de si beaux rêves d'indigence et de liberté au sein de ce bien-être qui m'oppressait, et dont j'aspirais toujours à sortir ! (...)Ne puis-je pas être courageuse et forte, moi qui n'ai dans les veines que du bon sang plébéien? (1959: 100-101)

C'est donc le voyage qui permet à Consuelo alias George Sand d'échapper au risque de renier ce qu'elle considère comme la meilleure partie d'elle-même, c'est-à-dire son sang plébeien, synonyme de courage et de liberté.

Une fois encore on voit que l'éloge du voyage n'est pas lié à un goût qui pourrait être une affaire purement personnelle et subjective, voire superficielle, -affaire de caractère voire de disposition physique ou physiologique. Il fait partie des revendications politiques et sociales de George Sand et il s'agit d'un choix libre, délibéré, car si l'on s'en tient aux circonstances de sa naissance, elle aurait pu tout aussi bien choisir une autre partie de son sang, le sang noble et aristocratique, qu'elle portait en elle également.

On sait en effet que George Sand, par ses origines, était si l'on peut dire les choses ainsi, composée de deux moitiés! Ce qui signifie en clair qu'elle était née de père noble et d'excellente famille alors que sa mère, de milieu tout à fait populaire, n'avait jamais évolué par rapport à cette appartenance ni dans sa culture ni dans ses moeurs. On a souvent montré comment $\mathrm{G}$. Sand s'est ainsi trouvée partagée voire déchirée entre ses deux milieux d'origine, entre la tradition aristocratique incarnée par sa grand-mère paternelle (son père étant mort accidentellement quand elle était fort jeune) qui vivait dans la belle demeure de Nohant et l'attachement affectif pour sa mère accru par la culpabilité de ne pas faire assez pour elle (d'autant que la grand-mère la tenait soigneusement éloignée).

17 Reprenant dans la fiction ce que George Sand elle-même avait vécu dans la réalité, Consuelo son personnage aurait pu céder à la tentation de s'intégrer à la très noble famille des Rudolstadt mais lorsqu'elle se trouve ramenée par la nécessité du voyage à la pauvreté de ses origines, elle en éprouve plaisir et fierté. Et l'on a vu que ce retour est aussi pour elle un retour à la figure maternelle, ce qui le charge d'une forte affectivité. Pour Consuelo comme pour George Sand, affronter l'épreuve du voyage est le meilleur moyen de se prouver à soi-même qu'on n'est pas enfermé dans la trop douce torpeur réservée aux privilégiés.

18 Le défi à relever est d'autant plus grand qu'elle est une femme, ce qui l'expose à beaucoup plus de dangers et de mésaventures funestes que si elle était un homme. George Sand croise les deux problématiques, celle du genre et celle de la classe sociale. Il apparaît pourtant, à lire les aventures de Consuelo, que dans les deux cas la difficulté est surmontable. On la voit en effet aux prises avec des risques bien réels mais elle s'en tire sans dégât pour sa vertu et son honneur. Et ces épisodes se terminent finalement à son avantage, contribuant à lui assurer une gloire qu'elle ne doit qu'à elle-même (elle va devenir par la suite une artiste célèbre et reconnue). 

champêtres ou paysans de George Sand qui date de 1846, c'est-à-dire deux ans avant celui qui est sans doute le plus connu, La Petite Fadette, (un roman que son auteur rattache explicitement aux événements de la Révolution de 1848). Lorsque commence l'action de ce conte qui ne dure qu'un court laps de temps, Marie est une jeune paysanne berrichonne de seize ans, issue d'un milieu très pauvre où les familles ne peuvent survivre qu'en utilisant la force de travail de leurs enfants; et la pauvre petite, si l'on ose dire, ne paye pas de mine, en ce sens qu'elle est menue, timide, et a presque l'air d'une enfant. Le sujet de l'histoire est vraiment le récit d'un voyage, même si celuici ne dure que deux jours et une nuit ; l'aller-retour qui nous est conté change non seulement la vie de deux êtres au moins mais plus profondément toute la manière d'être et de penser au sein d'une petite société rurale, qui est évidemment à l'image de beaucoup d'autres. Ce court voyage est une aventure dans un monde paysan où on ne quitte son village que très rarement et pour des raisons exceptionnelles, faciles à répertorier. L'un des deux voyageurs de cette histoire est Germain, paysan veuf de vingt-huit ans qui part faire connaissance avec une éventuelle seconde épouse, car personne dans son village ne lui convient et il lui faut bon gré mal gré se lancer dans une expédition plus difficile qu'il n'y paraît. La preuve en est qu'il se perdra en route, égaré par le brouillard et bientôt pris par la nuit. L'autre voyageuse est la petite Marie qu'on a confiée à Germain car elle va dans la même direction que lui pour se placer dans une ferme où l'on a besoin d'une servante. Elle non plus ne sait rien de cet endroit où elle devra passer au moins une année ni du chemin qui y conduit.

Cette obligation pour les paysannes pauvres d'aller se placer loin de chez elles, chez des maîtres plus fortunés est attestée historiquement. Mais dans La Mare au Diable, ce voyage devient pour George Sand l'occasion d'un hommage, d'un éloge, à l'honneur et à 
l'avantage de la femme voyageuse, et ce de différentes manières ou pour différentes raisons. Pendant tout ce voyage qui devient une aventure au sens plein du mot puisqu'il faut improviser une nuit à la belle étoile avec un enfant dont la présence n'était pas prévue, faire face au froid, à la faim et à la peur, et finalement à l'obligation de rebrousser chemin - la petite Marie ne cesse de révéler des qualités que personne n'aurait pu soupçonner en elle et surtout pas Germain. Parce qu'elle est femme, et pauvre, elle a appris à survivre dans les plus mauvaises conditions, ce qui implique de la prévoyance, de la sagacité et un grand nombre de savoir-faire, à la fois très simples et quasi miraculeux si on n'a pas eu l'occasion de les acquérir. Elle sait instinctivement prendre soin du petit enfant, elle est capable d'allumer du feu en pleine nuit dans l'humidité d'un sous-bois et de faire cuire une pièce de gibier sans le moindre ustensile -au demeurant totalement sobre elle-même alors que l'homme, Germain, est incapable de faire taire son appétit. Sans l'ombre d'un doute la supériorité de Marie sur son compagnon est éclatante lorsqu'il s'agit de faire face aux aléas du voyage. Germain le reconnaît et s'en émerveille, en sorte que ce voyage lui est une révélation : la femme qu'il cherchait n'est autre que la petite Marie et non cette riche paysanne grassement installée dans ses avantages, dont il s'éloigne aussitôt qu'il la connaît.

\section{Rapprochement des classes sociales} éclater ses qualités méconnues, c'est aussi le moyen de dépasser et même d'inverser le désavantage social qui la séparait de Germain. L'histoire se termine par leur mariage, improbable au début, et dont on devine qu'il sera pleinement heureux. C'est le voyage qui a rendu possible ce rapprochement des classes sociales qui est le grand rêve socialiste de George Sand, un rapprochement qui passe par la nécessaire révélation de la femme aux autres et à elle-même. La conclusion, toujours la même, exprime l'une des convictions les plus tenaces de George Sand: le terme de mobilité est d'abord à prendre au sens propre, il faut que la femme bouge, qu'elle sorte de son immobilité.

d'Isabelle Eberhardt, sans doute plus connue que les autres en Algérie puisqu'elle y a vécu les quatre ou cinq années les plus intenses de sa vie avant d'y mourir en 1904. Mais dire qu'elle a vécu en Algérie ne signifie nullement qu'elle s'y serait immobilisée, c'est au contraire le lieu qu'elle a choisi pour y vivre en accord avec son besoin de mobilité, lieu de nomadisme puisqu'il s'agit du sud et non du nord du pays qu'en revanche elle détestait. Sa mort prématurée dont il vient d'être question et la grande difficulté qu'elle éprouvait à faire publier ses écrits expliquent que l'on doive reconstituer ce que signifiait pour elle le nomadisme- à partir de ce qu'elle a cependant réussi à dire et en tout cas à manifester par ses choix de vie.

Isabelle Eberhardt (1877-1904) à partir de 1899, pendant 5 ans au moins, n'a cessé de voyager dans le nord de l'Afrique, et notamment dans le sud de l'Algérie, région qui jouissait encore à l'époque d'un statut un peu spécial, impliquant un moindre degré de colonisation, au sens où ce mot signifie un strict encadrement de l'espace et un contrôle exercé sur les éventuels déplacements de ses habitants. Isabelle Eberhardt voyage dans le sud algérien en tant que "grand reporter " (c'est ainsi que nous dirions les choses aujourd'hui) mais on ne saurait croire que ses incessants voyages dans ces 
immenses régions désertiques s'expliquent suffisamment par le besoin d'exercer cette profession. Celle-ci est l'effet d'un choix volontaire et assumé et sur lequel d'ailleurs elle s'est clairement exprimée.

\section{Opposition à la société coloniale et bourgeoise : nomadisme}

Pour Isabelle Eberhardt, voyager incessamment dans le sud algérien est le moyen qu'elle a trouvé de signifier son opposition à la société coloniale et bourgeoise installée dans le nord du pays. Il est incontestable que ce choix du nomadisme est chez elle la forme d'un rejet. Incontestable aussi que celui-ci devient pour elle de plus en plus clair et conscient, à mesure que les années passent et qu'elle avance dans sa connaissance du pays.

Les raisons qui ont poussé Isabelle Eberhardt à venir en Algérie puis à y rester sont toutes personnelles : elle s'était convertie à l'islam, qui l'aidait à soulager son angoisse existentielle; d'autre part l'Algérie était devenue pour elle une sorte de substitut à sa mère bien-aimée, qui y était morte et enterrée. De plus, elle s'était sentie très atypique et non conforme dans les milieux littéraires français, d'où son choix de les fuir, par impuissance ou dénégation. Enfin, il est certain que son tempérament, de ceux qu'on dit instables, la pousse au nomadisme : les longues chevauchées dans le désert sont ce qu'elle aime par dessus tout et l'on pourrait se contenter - ou presque- de dire que cela fait partie de sa personnalité. Ce serait une première lecture de son œuvre ${ }^{6}$ (publiée pour l'essentiel à titre posthume, bien après sa mort accidentelle dans la crue de l'oued d'Aïn Sefra en 1904).

Il est pourtant évident aussi qu'une orientation idéologique précise se dégage de ces textes assez clairement. Leur auteure ne partage en aucune façon le rêve occidental d'améliorer le mode de vie des "Indigènes » et d'y introduire le progrès. A l'inverse, elle dénonce violemment l'entreprise coloniale dont elle ne voit que les aspects négatifs, et fuit tous les lieux où celle-ci se manifeste. En sorte que l'on peut certainement parler à cet égard de son anticolonialisme, fondamentalement opposé aux idéaux démocratiques proclamés ou affichés par le gouvernement français de la Troisième République (faut-il rappeler que sur le terrain ils n'ont cessé d'être trahis ?).

\section{Contre l'installation : éloge du dénuement}

31 Le voyage pour Isabelle Eberhardt est le contraire de ce qu'elle considère comme une installation dans la médiocrité petite-bourgeoise qui lui paraît le fait de la plupart des colons, en tout cas ceux qu'elle a l'occasion de connaître. Incontestablement, son attitude est méprisante mais elle est prête à payer le prix fort pour avoir droit à ce mépris. On peut parler chez elle d'une attitude aristocratique, peut-être par fidélité à son ascendance maternelle (sa mère appartient à la noblesse russe d'origine allemande) mais surtout par choix personnel d'une sorte d'élitisme qui l'éloigne de tout ce qui lui paraît une satisfaction niaise due à la position trop facilement dominante assurée par la hiérarchie coloniale. Aristocratisme qui explique sans doute ses affinités avec Lyautey: la société tribale arabe ou arabo-berbère des territoires sahariens représente à peu près leur idéal social en ce qu'elle est en effet aristocratique-aristocratique et misérable comme l'est Isabelle elle-même. 

peut désigner comme choix du voyage, ou choix du nomadisme) va parfaitement de pair avec une pauvreté et un dénuement presque inconcevables. Elle mange rarement à sa faim, compense le manque avec de l'alcool et du kif, soumettant au même régime son compagnon le spahi Slimène Ehnni. Ils ne possèdent rien, n'ont aucun lieu stable et aucun des moyens auxquels a recours l'humanité ordinaire pour se "sécuriser" (expression très employée aujourd'hui, qu'Isabelle Eberhardt ne connaissait pas mais qu'elle aurait sans doute détestée !)

Voyons concrètement, à partir de certains de ses écrits, comment se passe la vie matérielle d'Isabelle Eberhardt lorsqu'elle est en voyage. Sans un sou vaillant, elle dort souvent à la belle étoile, à flanc de dune. Mais elle connaît aussi ce qu'elle appelle « la volupté des logements de hasard ». C'est le cas lorsqu'elle trouve l'hospitalité sous une tente ou dans un ksar7. Voici par exemple quelques mots empruntés à la description qu'elle donne de l'un d'entre eux: "une chambre fruste en très vieille toub ${ }^{8}$ grise, sorte d'antre aux parois irrégulières, au plafond bas en tiges de palmes noircies, toutes gondolées " (2003 : 44). Dans ces lieux, elle passe d'interminables veillées à entendre ce que les ksouriens lui racontent, trop heureux de trouver un auditeur attentif en la personne de Si Mahmoud, le nom d'homme qu'elle s'est donné pour ses voyages. Au gré de ce qu'on lui suggère, elle se rend dans telle ou telle zaouia ${ }^{9}$, ou se contente de regarder les lieux et les gens autour d'elle, d'un regard d'écrivain; jusqu'au moment où elle se remet en route, toujours aussi pauvre que ceux qu'elle côtoie. du sud, la question de l'argent ne se pose même pas, elle vit entièrement de l'hospitalité arabe qui est pour elle une sorte d'évidence, et dont l'absence lui rend très difficile voire insupportable la vie dans le nord du pays. En fait, ce à quoi elle aspire est le mode de vie et "la sagesse des fakirs et anachorètes musulmans» (2003: 251). Et c'est évidemment une déclaration très impressionnante par ce qu'elle implique. Point n'est besoin d'y insister. On aura compris qu'il y a, partiellement sans doute, dans cette pratique du voyage et de l'errance, un aspect auto-destructeur. Tendance ou pulsion confirmée dans le cas d'Isabelle puisqu'elle entraîne effectivement sa fin rapide, dite accidentelle, en 1904). Nous sommes là aux antipodes de l'optimisme sandien, et même du militantisme épuisant dont meurt Flora Tristan, par volonté de se battre jusqu'au bout pour ses convictions. Isabelle Eberhardt est plongée dans les circonstances très particulières de l'entreprise coloniale, à laquelle elle ne saurait adhérer. Ces circonstances lui interdisent de croire au progrès social, c'est pourquoi elle se réfugie dans le voyage pour échapper à ce prétendu progrès dont se flatte la politique officielle et qu'elle prétend développer.

\section{Voyage et émancipation}

Quoi qu'il en soit de leurs différences, ces trois exemples de "femmes qui voyagent » apportent la preuve de l'importance qu'elles accordent à cette activité et de la signification qu'elles lui confèrent. On croit pouvoir affirmer que, du début à la fin du $19 \mathrm{e}$ siècle, le voyage a été pour les femmes un des grands moyens d'émancipation et d'affirmation d'elles-mêmes. 


\section{BIBLIOGRAPHIE}

EBERHARDT, Isabelle, Au pays des sables, (nouvelles), Editions Joëlle Losfeld, Paris, 2002.

EBERHARDT, Isabelle, Journaliers, (journal intime), Editions Joëlle Losfeld, Paris, 2002.

EBERHARDT, Isabelle, Sud Oranais, (notes de voyage), Editions Joëlle Losfeld, Paris, 2003.

SAND, George, Consuelo, La Comtesse de Rudolstadt, Paris, Classiques Garnier, 3 volumes, 1959.

SAND, George, La Mare au diable, Livre de Poche LGF, 1973.

TRISTAN, Flora, Nécessité de faire un bon accueil aux femmes étrangères, Paris, L'Harmattan, 1988.

TRISTAN, Flora, Les Pérégrinations d'une paria, Paris, La Découverte, 1979.

\section{NOTES}

1. On aura l'occasion de voir que cet ordre qui est chronologique est aussi celui d'une évolution et devait donc être respecté.

2. Racontée de manière assez fidèle par Mario Vargas Llosa dans Le Paradis-un peu plus loin, Gallimard, 2003

3. Les passages évoqués se trouvent dans le 2ème volume.

4. Il y a chez George Sand de nombreux éloges rendus aux populations nomades, alors même qu'il ne s'agit que de groupes résiduels. C'est par exemple le cas des Bourbonnais, qu'elle oppose aux Berrichons sédentaires dans Les Maîtres sonneurs.

5. Préface et commentaires de Pierre de Boisdeffre.

6. Editions Joëlle Losfeld, Paris : Au pays des sables, (nouvelles), 2002. Journaliers (journal intime), 2002. Sud Oranais (notes de voyage), 2003.

7. Nom donné au village saharien. Les habitants sont les ksouriens.

8. Argile séchée

9. Siège d'une confrérie

\section{RÉSUMÉS}

Cet article est consacré à trois femmes du $19^{\mathrm{e}}$ siècle, Flora Tristan, George Sand et Isabelle Eberhardt qui ne sont pas de celles qu'on pourrait appeler des voyageuses professionnelles, mais plutôt des femmes que les circonstances ont amenées à voyager (Flora Tristan), ou qui ont réfléchi aux particularités du voyage dans la vie des femmes (George Sand), ou qui ont «nomadisé » faute de pouvoir supporter la vie sédentaire (Isabelle Eberhardt). Etant en même temps écrivaines, elles ont eu le désir et la capacité de transmettre leur expérience du voyage. Il nous apparaît grâce à elles que le voyage a été pour les femmes du 19e siècle un grand moyen d'émancipation et d'affirmation d'elles-mêmes. 
This paper is dedicated to three women of the $19^{\text {th }}$ century, Flora Tristan, George Sand and Isabelle Eberhardt. None of them chose travelling as her main occupation, Flora Tristan had to go abroad because of some special circonstances in her life, George Sand wanted to consider the main role of travel in a woman's life, Isabelle Eberhardt was unable to stay quietly among the ladies of colonial Algeria. Being at the same time writers, they had the desire and the capacity to transmit their experience of travelling.Thanks to them, we understand that through travelling, women of the $19^{\text {th }}$ century found a way to free and assert themselves.

\section{INDEX}

Mots-clés : voyage, femmes, mobilité, nomadisme, émancipation

الترحال, نساء, الحركية, البداوة, تحرّفهرس الكلمات المفتاحية:

Keywords : travel, women, motion, wandering, freedom

\section{AUTEUR}

DENISE BRAHIMI

Université Paris VII-Denis Diderot 